\title{
Accuracy Enhancement of Position Estimation using Adaptive Kalman Filter and Map Matching
}

\author{
Youngwan Cho and Hyunkyu Choi \\ Dept. of Computer Engineering, Seokyeong University \\ 124 Seo-kyeong-Ro, Seong-buk-Gu, Seoul, 136-704, Korea \\ ywcho@skuniv.ac.kr,andlsguqror@nate.com
}

\begin{abstract}
It is an essential factor in automatic navigation systems of ground vehicles, railroad and aviation fields which require high stability to detect the global position of vehicles with credibility and accuracy. This paper proposes an approach to estimate the global position of vehicles moving in the predefined path with a set pattern. Because the information of location received by GPS is included various errors, the errors are minimized using GPS error filter and map matching to obtain correct information. The velocity profile is one of key information for the system model of moving vehicles. We propose an estimation scheme using adaptive Kalman filter where the system model is derived from the moving pattern of vehicles. Since there is high probability that the position obtained from the adaptive Kalman filter is deviated from actual road and/or railway path, we remove the error by using map matching technique. The proposed scheme was experimented in position detection of train moving along predefined section of railway.
\end{abstract}

Keywords: Position estimation, Kalman Filter, Moving pattern, Map matching, GPS error filter

\section{Introduction}

Generally, in railroad and aviation fields which require high stability systems, detecting global position with credibility and accuracy is an essential factor. When a train system is traveling at $100 \mathrm{~km} / \mathrm{h}$ on the railway, it has a dynamic characteristic such that it requires a minimum of $1 \mathrm{~km}$ for braking distance. The train control system introduced which has an objective to prevent the train's rear end and collision, has an objective to control space to guarantee braking space. In order to achieve this object, an accurate positioning of the train's location is a prerequisite. From the dynamic characteristic which requires a long braking distance, when the train is operating at normal conditions, it can be assumed that the train will be operated at specific movement patterns to maintain safety distance and operate efficiently at the same time. Position data obtained from sensors attached to real trains were used to approximate movement patterns. The approximated movement patterns were applied to Kalman filter [8] and depending on the global position, the Kalman filter was designed to change adaptively to the state transition matrix. Map matching is a technique which assumes the vehicle is always operating on top of the road and railroad and the estimated position is matched to the actual road in order to improve the position. The above technique is used to revise inaccurate position sensor data to make it closer to the actual position. The point-to-point, point-to-curve and fuzzy rule based techniques are typical approaches [1]. 


\section{System Model}

An adaptive Kalman filer is used for position detection technique which works in the process shown in Figure 1. The inaccurate position sensor data is revised by the map matching technique. The revised position data is input to the noise filter. In this paper, latitude longitude directional moving patterns are devived from the velocity and position data of the train moving on a specific section. The approximated moving pattern is applied to the state transition matrix of the Kalman filter [8] to estimate the position and velocity. The estimated position is used for the map-matching technique to detect the final position.

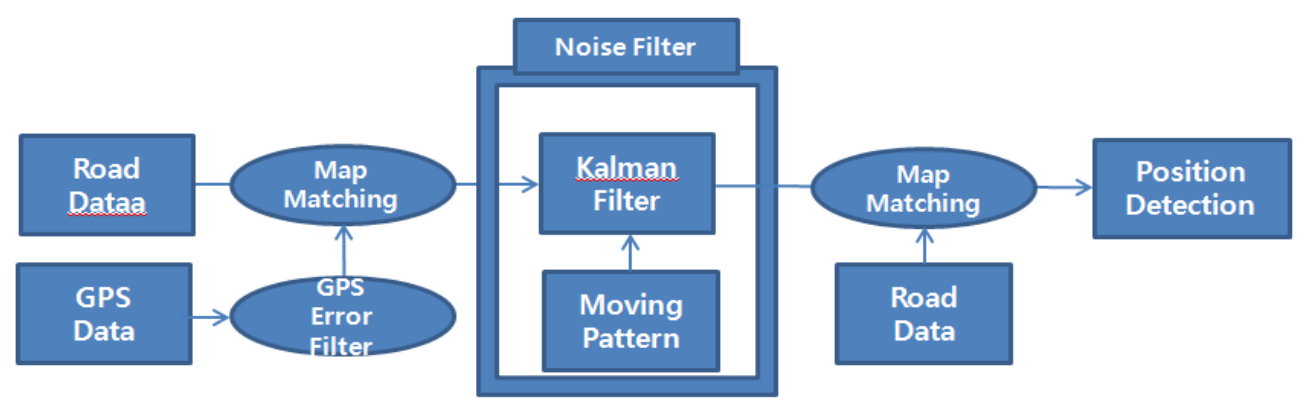

Figure 1. Block Diagram of Position Detection

When starting and stopping point for railroad cars are designated, it has a characteristic such that the travel course between the two points are always fixed. This means, all points in between the two points (starting and stopping point) have to be passed by in any situation. Figure 2 shows map of railway section. The experimental position data was obtained from GPS on the train operated in this section.

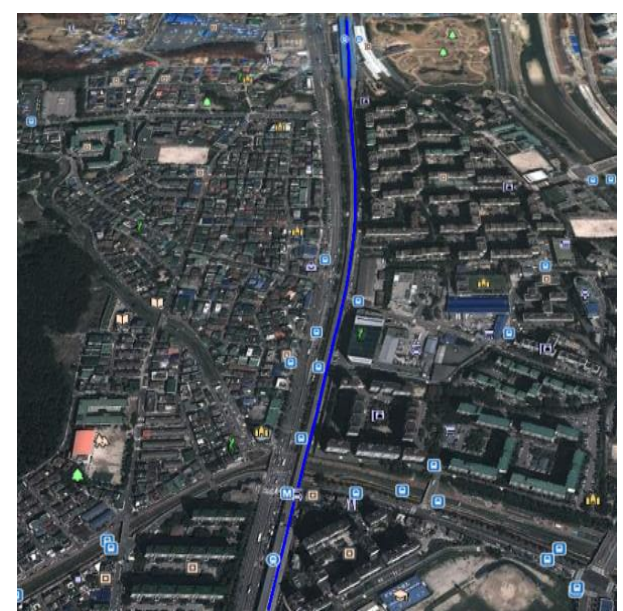

\section{Figure 2. Dobong $\leftrightarrow$ Dobong Mountain Section}

As shown in the figure2, the train operated in Dobong $\leftrightarrow$ Dobong Mountain section must pass by all rail way between the two points. In this paper, the actual position data for trains passing the section of Dobong $\leftrightarrow$ Dobong Mountain was obtained and this data was used to approximate the train moving pattern. 


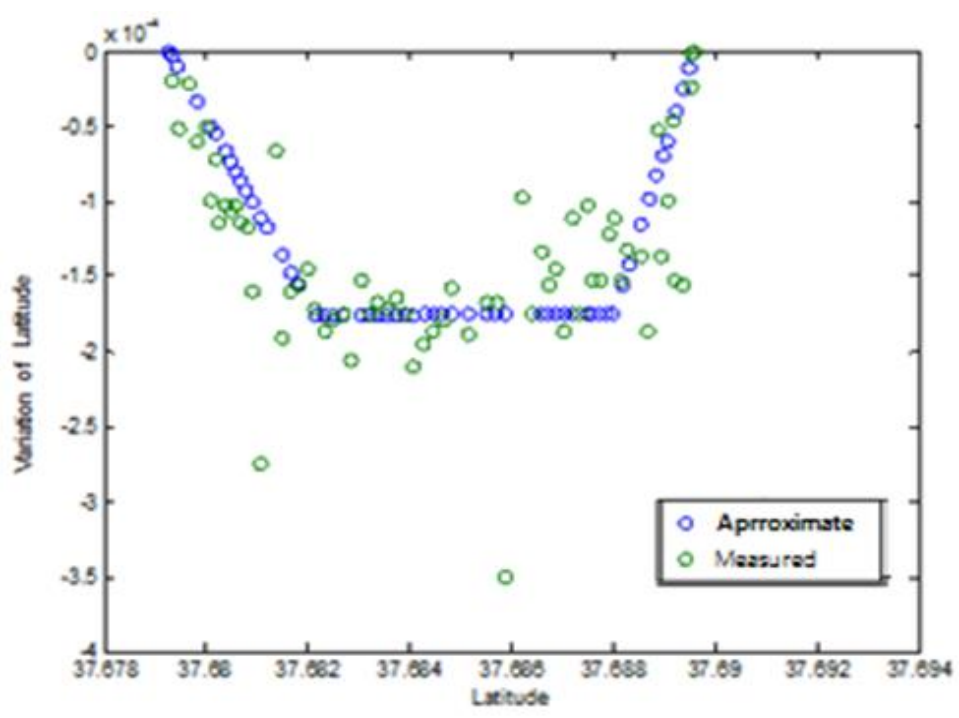

Figure 3. Latitude Directional Moving Pattern Approximation

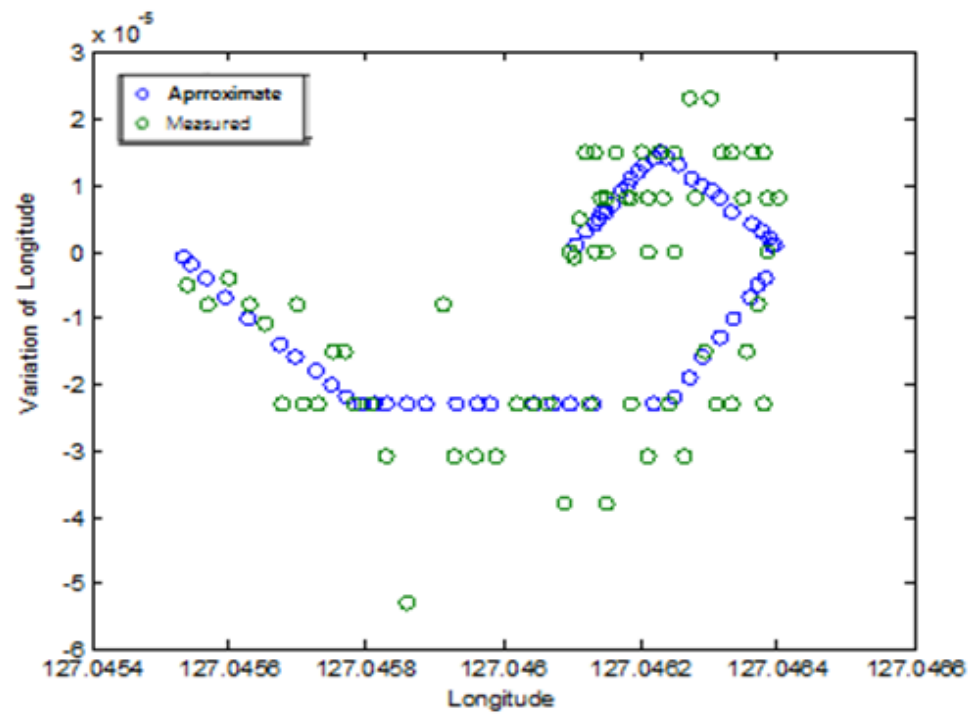

Figure 4. Longitude Directional Moving Pattern Approximation

Figure 3 and Figure 4 show velocity changes in terms of latitude and longitude directional position on the Dobong $\leftrightarrow$ Dobong Mountain section, respectively. The velocity is change rate of longitude and latitude position on time and the equation for the velocity is given in (1).

$v=\frac{s_{n}-s_{n-1}}{t_{n}-t_{n-1}}$

where, $\mathrm{v}$ is velocity, $\mathrm{s}$ is the position acquired from the sensor (Map matched longitude and latitude position), $\mathrm{t}$ is the time acquired from the sensor, and $\mathrm{n}$ is the number of samples.

\section{Adaptive Kalman Filter}

The adaptive Kalman filter is designed based on the Kalman filter [8]. When the characteristics of a system change with time and sensor signal measuring the output of the system contains a lot of noise, the Kalman filter is used to obtain noiseless signal by 
estimating the system and compensating the measured signal. The data obtained from a position sensor which the accuracy cannot be trusted is used in the map matching technique to match with actual position of rail way and reduce noise. The matched position data is used for the input of the adaptive Kalman filter. The Kalman filter composition proposed in this paper is as shown in equation (2).

$x_{k+1}=A x_{k}$

where, $A=\left[\begin{array}{cc}1 & \Delta t \\ 0 & \alpha\end{array}\right]$ and $x=[s v]^{T}$.

Equation (2) represents the state equation of the Kalman filter, where A is the state transition matrix , $x$ is the state variable, $\mathrm{k}$ is the number of sample and $\Delta \mathrm{t}$ is the elapsed time between sample $\mathrm{k}+1$ and $\mathrm{k}$. The $\alpha$ is an estimated acceleration composite obtained from approximating the moving pattern. In equation (2), the longitude and latitude directional position is represented as $s$ and the velocity is represented as $v$. In this paper, the Kalman filter estimates longitude and latitude position and velocity independently. In order to find the estimated acceleration composite $\alpha$, the approximated moving pattern obtained previously is used. As mentioned in the introduction, if a vehicle is operated at a specific moving pattern in a specific course, the velocity at a point in the specific course can be estimated.

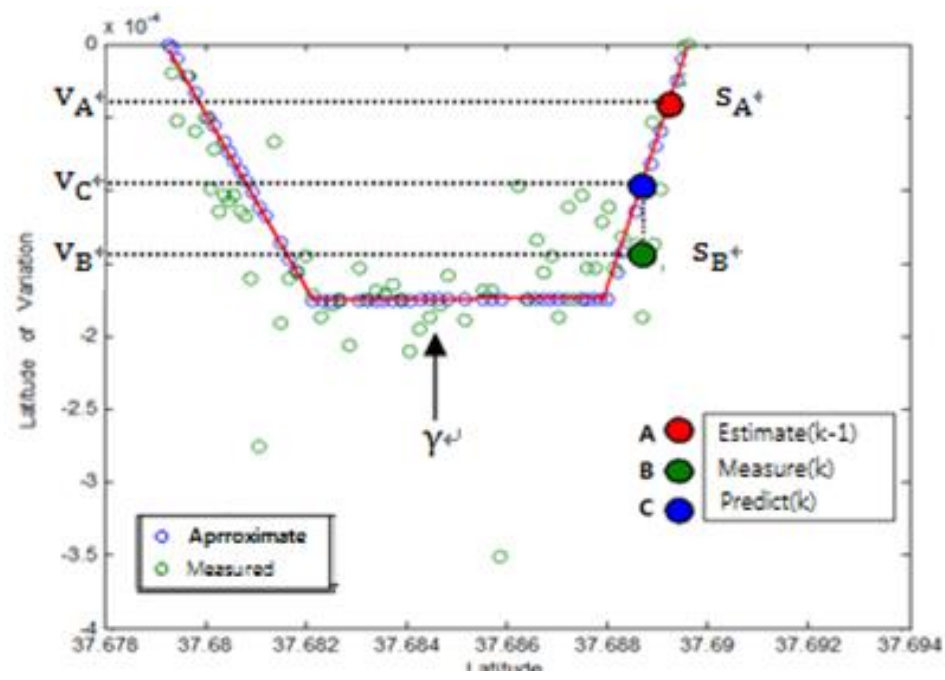

Figure 5. Moving Pattern to Find $\alpha$

In Figure 5, the variables are assumed as: $\mathrm{s}_{\mathrm{A}}$ the estimated position at the sampling time $\mathrm{k}-1, \mathrm{v}_{\mathrm{A}}$ the estimated velocity, $\mathrm{s}_{\mathrm{B}}$ the measured position at sampling time $\mathrm{k}$ and $\mathrm{v}_{\mathrm{B}}$ as the measured velocity. The $\mathrm{x}$-axis stands for the position and the $\mathrm{y}$-axis stands for velocity. The approximated moving pattern value is $\gamma$ and estimated velocity is $v_{C}$. The estimated acceleration composition $\alpha$ is as shown in equation (3).

$$
\alpha=\frac{v_{C}}{v_{A}}
$$

where, $\alpha$ plays a role of changing the estimated velocity in state transition matrix depending on the moving pattern. Therefore, the estimated acceleration composite $\alpha$ is a parameter to find estimated velocity. In Figure 5 , the measured velocity $v_{B}$ which is not used to find $\alpha$ is used in the Kalman filter update part to detect the estimated velocity. A measurement equation is required to utilize the Kalman filter. The measured equation used in this paper is shown in equation (4).

$z_{k}=H x_{k}, H=\left[\begin{array}{ll}1 & 1\end{array}\right]$ 


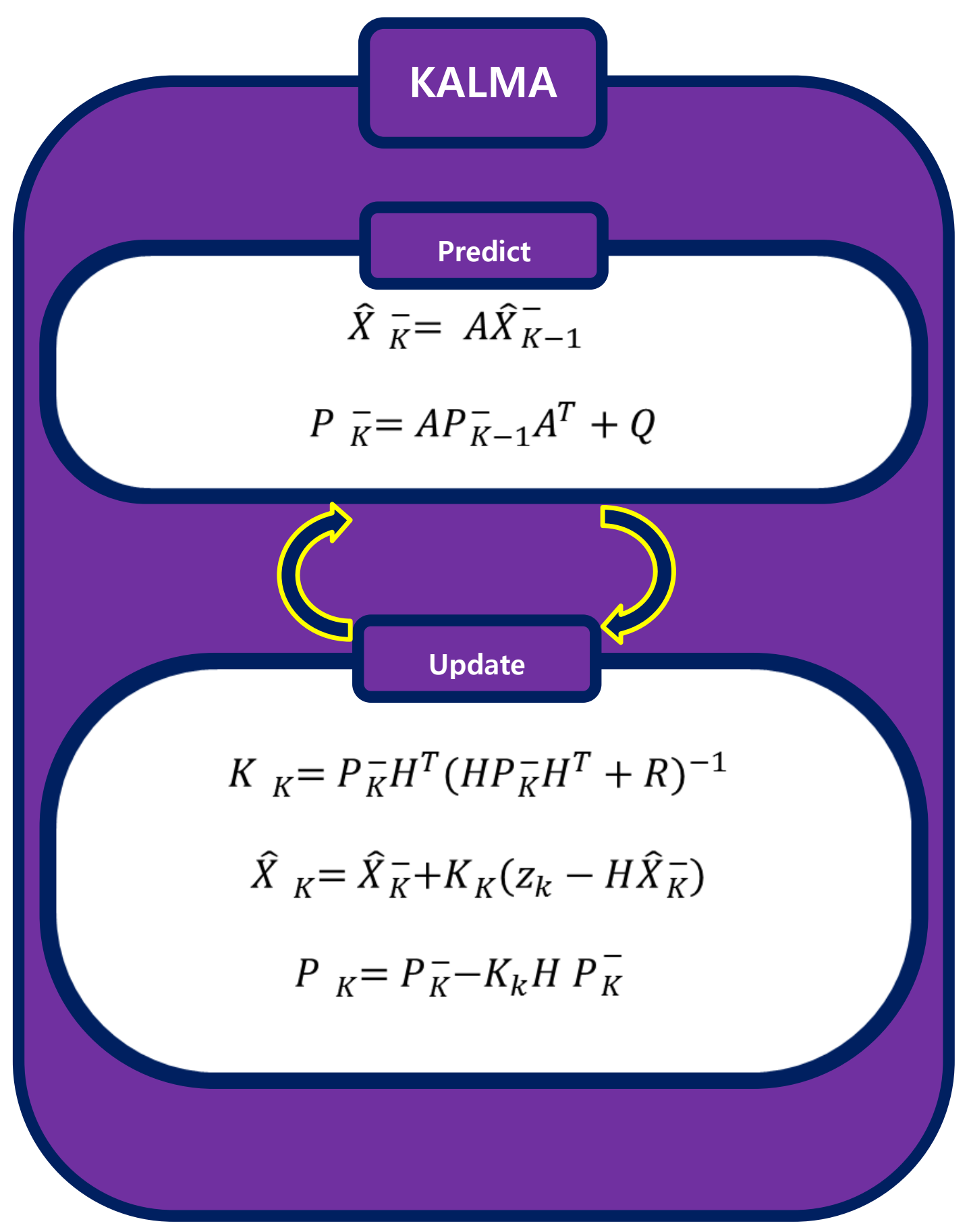

Figure 6. Kalman Filter's Process

The state variables in the measured equation $x=\left[s^{\prime} v^{\prime}\right]^{T}$, the position data in map matching is designated to $s^{\prime}$ and measured velocity $v_{B}$ in Figure 5 is designated to $v^{\prime}$. When the model composition is completed, the Kalman filter process is performed repeatedly as shown in Figure 6.

A method to automatically separate the start and stop of the position detection technique using the adaptive Kalman filter within a specific region, the closeness of the vehicle from the start and stop point are used as a standard. Figure 7 and 8 are the obtained data from a vehicle operating through the Dobong $\leftrightarrow$ Dobong Mountain section (the same experiment was repeated twice). $\mathrm{C}$ is the time taken for the train 
to stop at Dobong station and leave again. $\mathrm{B}$ is the time taken to reach the destination. The leaving time $\mathrm{C}$ and arriving time $\mathrm{B}$ are different in Figure 7 and 8 but the position of $\mathrm{C}$ and $\mathrm{B}$ are almost the same. Therefore, the position data from the sensor are continuously obtained before the train leaves and the Kalman filter is operated from the point the position data leaves the starting point. The Kalman filter is terminated when the estimated position data from the filter arrives at the destination. A is the poor data obtained from the sensor while the train is operated.

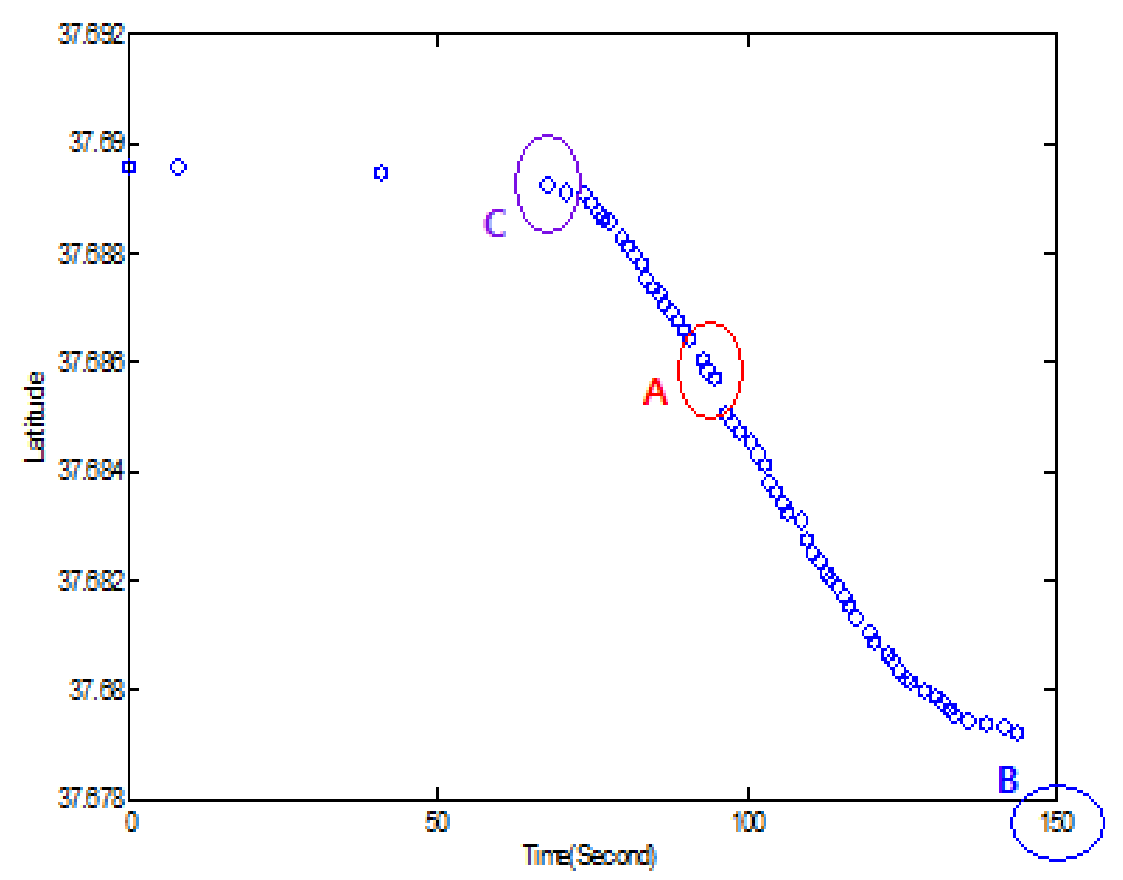

Figure 7. Change in Latitude Depending on Time in Dobong $\leftrightarrow$ Dobong Mountain Section 1

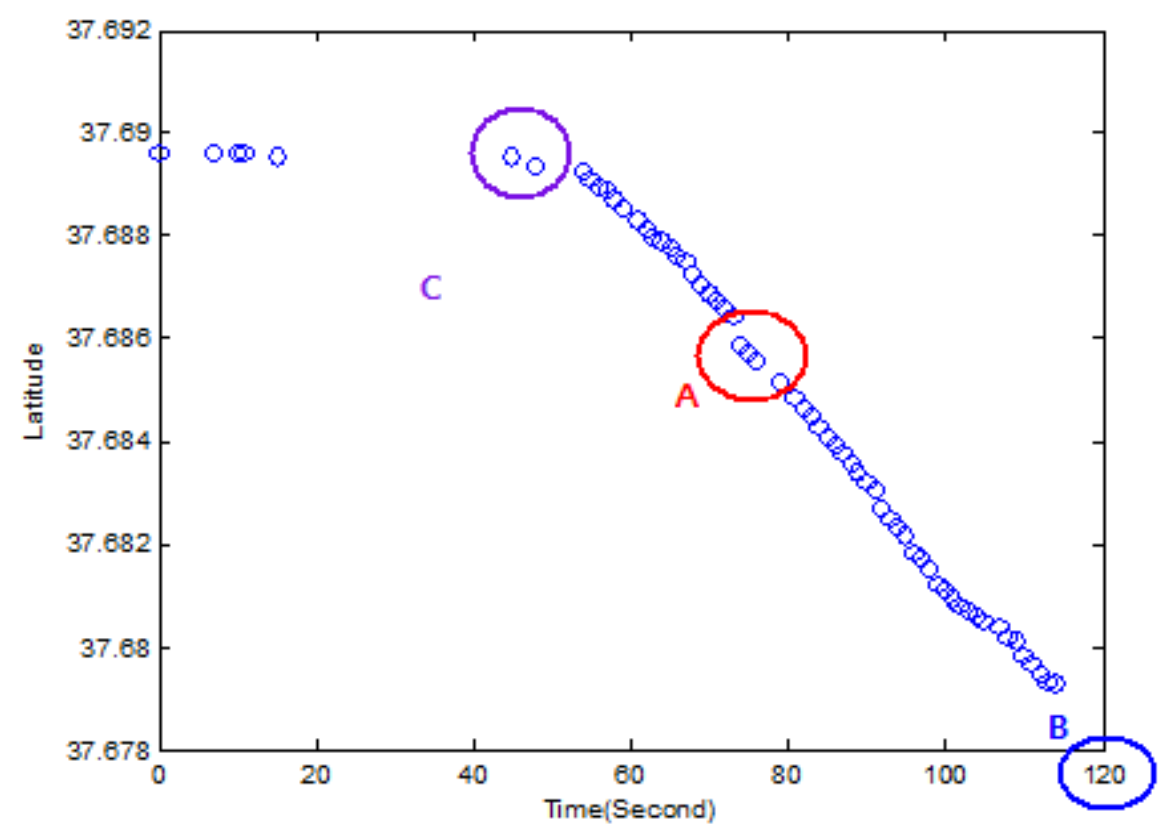

Figure 8. Change in Latitude Depending on Time in Dobong $\leftrightarrow$ Dobong Mountain Section 2 


\section{GPS Error Filter}

Generally, GPS data includes error which can be classified into bias error and white noise error. The information of location received by GPS can differ by actual location from few meters to few tens of meters because the information includes biased error and white noise [7].
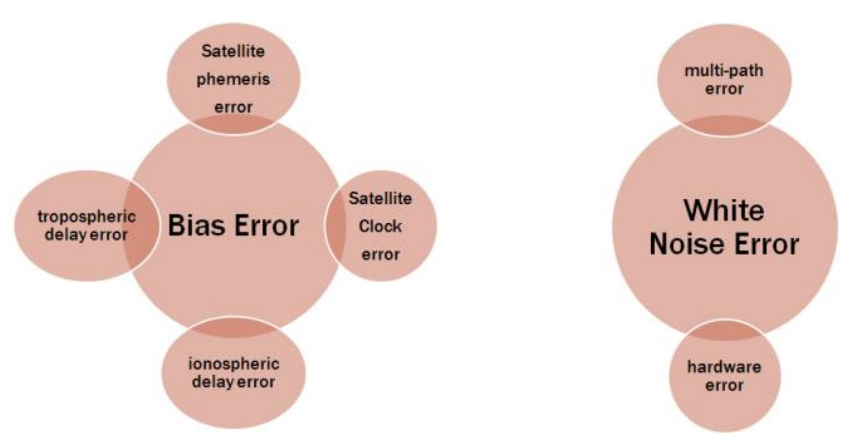

Figure 9. GPS Error

The use of information of location removed GPS error is more reasonable to estimate the actual location than the use of information of location included GPS error. Therefore, in this paper, GPS error is modeled and estimated using Kalman Filter in order to remove it. The GPS error is expressed as equation (5).

$e(k)=e_{\text {bias }}(k)+e_{\text {white }}(k)$

The $\mathrm{e}(\mathrm{k})$ means the GPS error at time $\mathrm{k}$ in equation $(5), e_{\text {bias }}(\mathrm{k})$ is bias error at time $\mathrm{k}$, and $e_{\text {white }}(\mathrm{k})$ is white noise error at time $\mathrm{k}$. Because bias error is generated by meteorological factors and satellite problem, equation (5) can be approximated to equation (6).

$e(k) \approx e_{\text {white }}$

The state equation of GPS error filter is given in equation (7).

$x_{k+1}=A x_{k}+w, x=\left[\begin{array}{c}S_{n} \\ S_{e} \\ V_{n} \\ V_{e} \\ \Delta s_{n} \\ \Delta S_{e}\end{array}\right], A=\left[\begin{array}{cccccc}1 & 0 & 1 & 0 & 0 & 0 \\ 0 & 1 & 0 & 1 & 0 & 0 \\ 0 & 0 & 1 & 0 & 0 & 0 \\ 0 & 0 & 0 & 1 & 0 & 0 \\ 0 & 0 & 0 & 0 & 1 & 0 \\ 0 & 0 & 0 & 0 & 0 & 1\end{array}\right], w=\left[\begin{array}{c}0 \\ 0 \\ w_{v n} \\ w_{v e} \\ w_{\Delta S n} \\ w_{\Delta S e}\end{array}\right]$

State variable $S_{n}$ and $S_{e}$ are north-south direction(latitude) and east-west direction (longitude) of the location, respectively. The $V_{n}$ and $V_{e}$ are north-south and east-west direction of the velocity, respectively. And the $\Delta \mathrm{S}_{\mathrm{n}}$ and $\Delta \mathrm{S}_{\mathrm{e}}$ are north-south and eastwest direction of the GPS error, respectively. The Relation between position of the state variables and the position of the measurement is as equation (8).

$Z_{S n}(k)-Z_{\Delta S n}(k)=S_{n}(k)+v_{\Delta S n}$

$$
Z_{S e}(k)-Z_{\Delta S e}(k)=S_{e}(k)+v_{\Delta S e}
$$

$\mathrm{Z}_{\mathrm{Sn}}(\mathrm{k})$ and $\mathrm{Z}_{\mathrm{Se}}(\mathrm{k})$ are measurement values, north-south and east-west direction nal position received by GPS at time $\mathrm{k} . \mathrm{Z}_{\Delta \mathrm{Sn}}(\mathrm{k})$ and $\mathrm{Z}_{\Delta \mathrm{Se}}(\mathrm{k})$ are the distance difference between the north-south and east-west of the positions received by the GPS and the north- 
south and east-west of the positions resulted by map matching. The $\mathrm{v}$ represents noise on the GPS sensor which follows the distribution of $N\left(0, R_{\Delta S n}\right)$. The measurement equation of GPS error filter is as follows.

$Z_{k}=H x_{k}+v, \quad H=\left[\begin{array}{llllll}1 & 0 & 0 & 0 & 0 & 0 \\ 0 & 1 & 0 & 0 & 0 & 0 \\ 0 & 0 & 1 & 0 & 0 & 0 \\ 0 & 0 & 0 & 1 & 0 & 0 \\ 0 & 0 & 0 & 0 & 1 & 0 \\ 0 & 0 & 0 & 0 & 0 & 1\end{array}\right], \quad v=\left[\begin{array}{c}v_{S n} \\ v_{S e} \\ v_{v n} \\ v_{v n} \\ v_{\Delta S n} \\ v_{\Delta S e}\end{array}\right]$

\section{Experiment and Result}

In order to determine the error between the actual position and the GPS position, fixed to the specific absolute position. Error is the distance between the actual position and the GPS position. Here, the actual position of the GPS, which is fixed, is referred to as 'center point'. Figure 10 is a representation of the map errors in data obtained using the GPS error filter and general matching data.

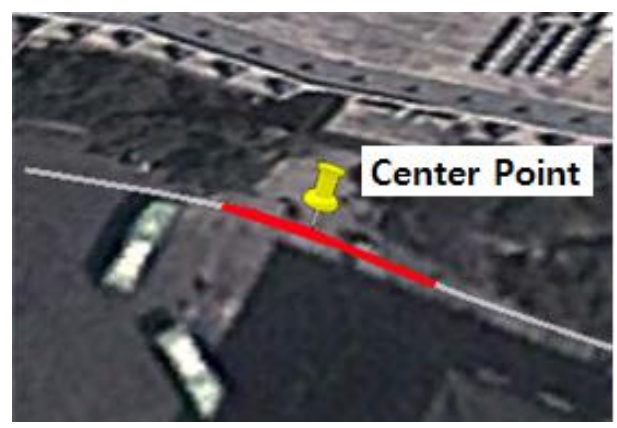

(a) Point-to-Curve Matching

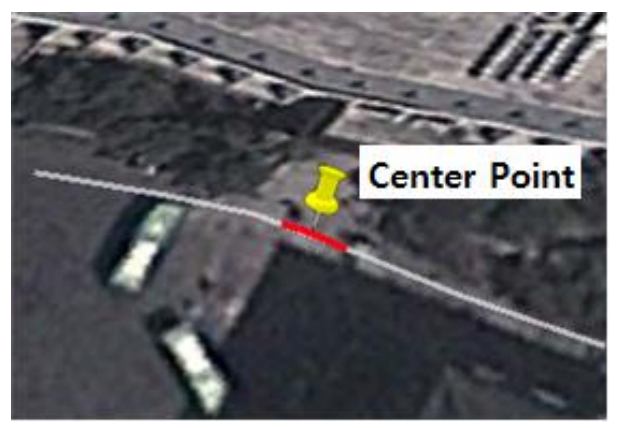

(b) Matching Using GPS Error Filter

Figure 10. Comparing the General Matching and the Matching using GPS Error Filter

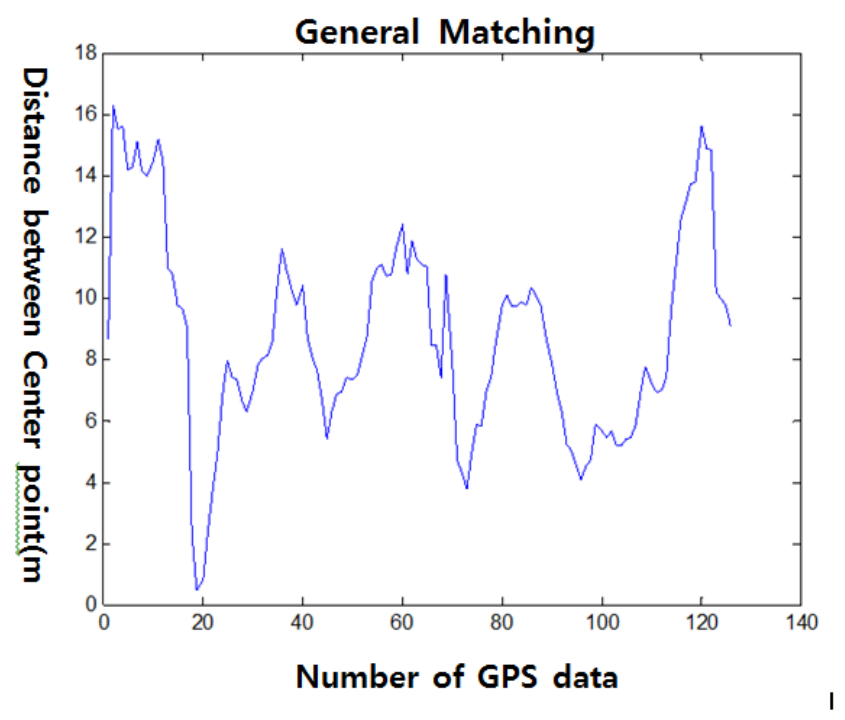




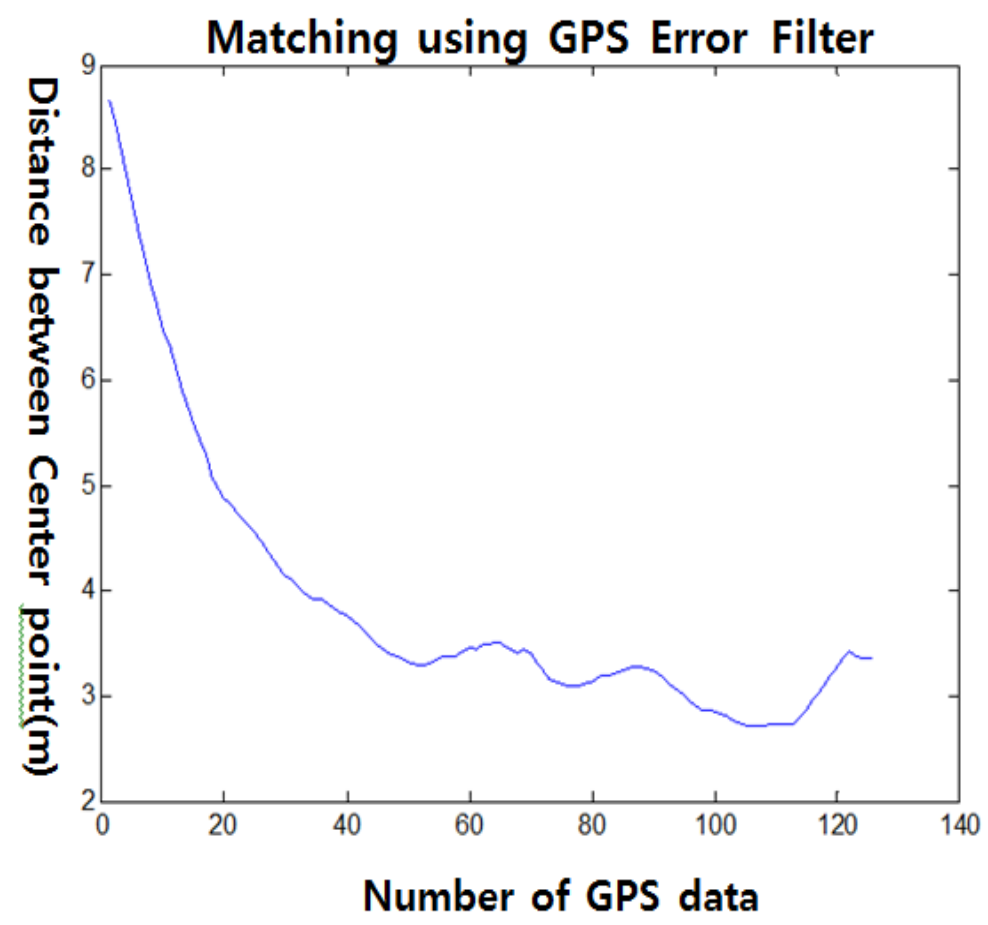

Figure 11. Distance Between Center Point

In Figure 10, the gray line represents the matching path and the red line on the path is matched data, where marking point means center point. The error of the applied GPS error filter data is smaller than general matching data. Figure 11 shows the variation of position error with time for the cases of point to curve matching and GPS error filter matching. Table 1 shows the average of the distance from the center point about the applied GPS error filter data and general matching data.

Table 1. Average Distance between Center Point

\begin{tabular}{|c|c|}
\hline & Average distance between center point \\
\hline General matching & $8.7679 \mathrm{~m}$ \\
\hline Matching using GPS error filter & $3.8801 \mathrm{~m}$ \\
\hline
\end{tabular}

The adaptive Kalman filter which uses moving pattern is a position estimation filter for a set pattern movement in a limited situation traveling a specific section. In this paper, the position estimation schme proposed in this paper is applied to a moving train operated on Dobong $\leftrightarrow$ Dobong Moutaion section. In Figure 12, the path A means the position data obtained from the position sensor and the path B means the actual position of Dobong $\leftrightarrow$ Dobong Mountain section. From the Figure 12, we can note that the latitude directional noise could not be found but the longitude directional noise could be found. As shown in Figure 13, to detect the revised position from the unreliable sensor data, the Kalman filter was used and the results are shown in Figure 13 and 14. 


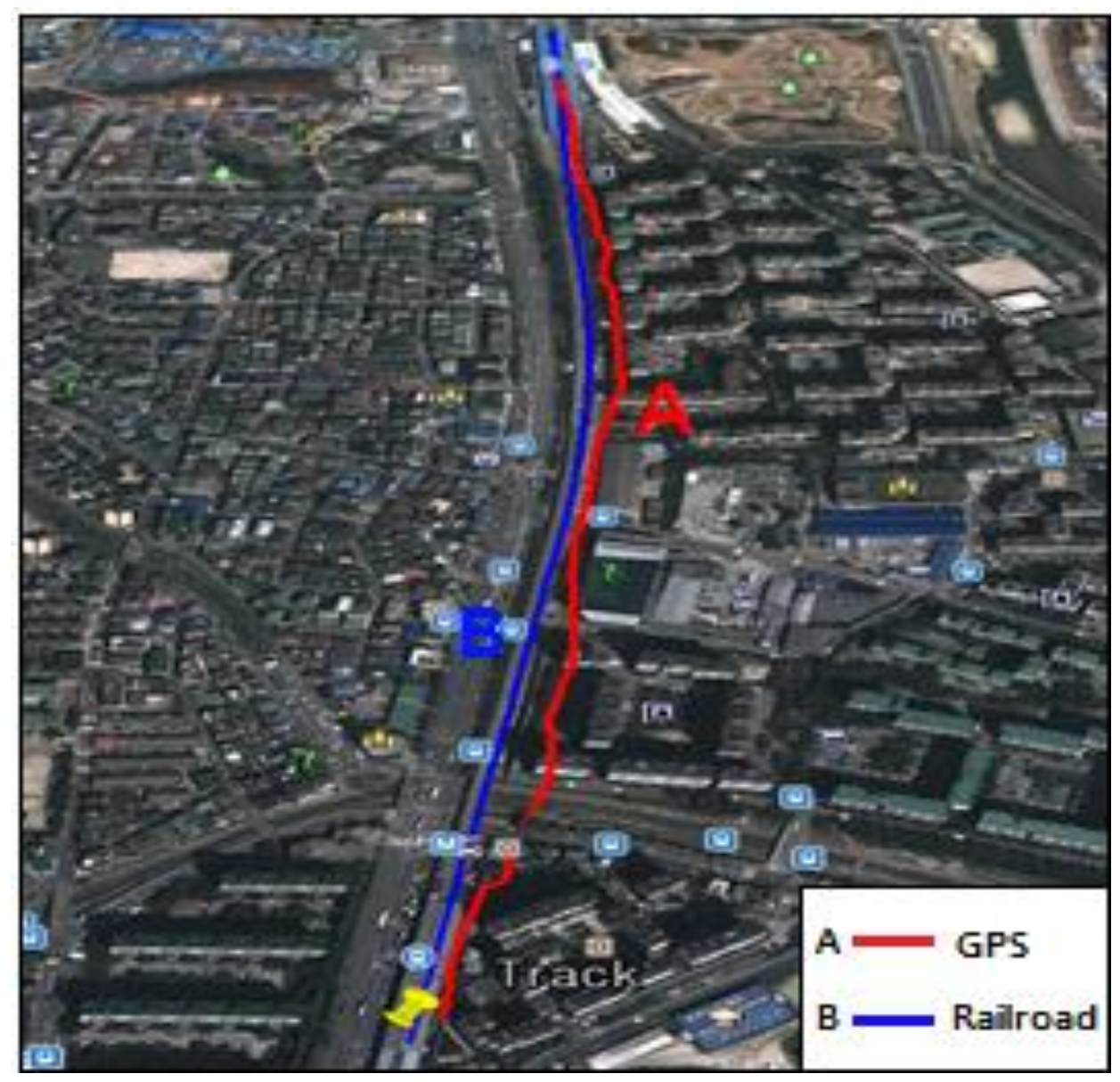

Figure 12. Dobong $\leftrightarrow$ Dobong Mountain Section GPS Course

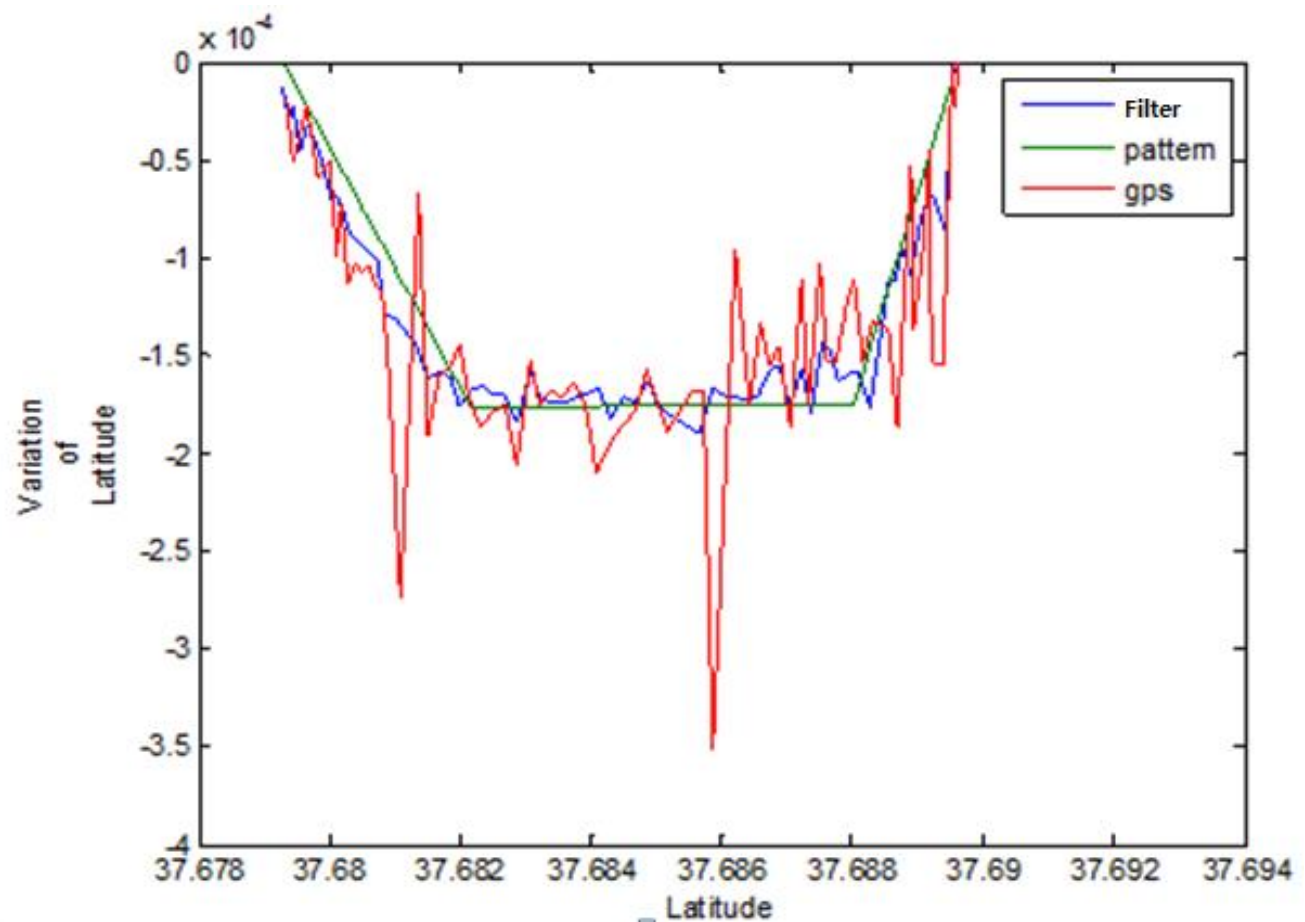

Figure 13. Velocity Results Depending on Latitude Passed through the Kalman Filter 


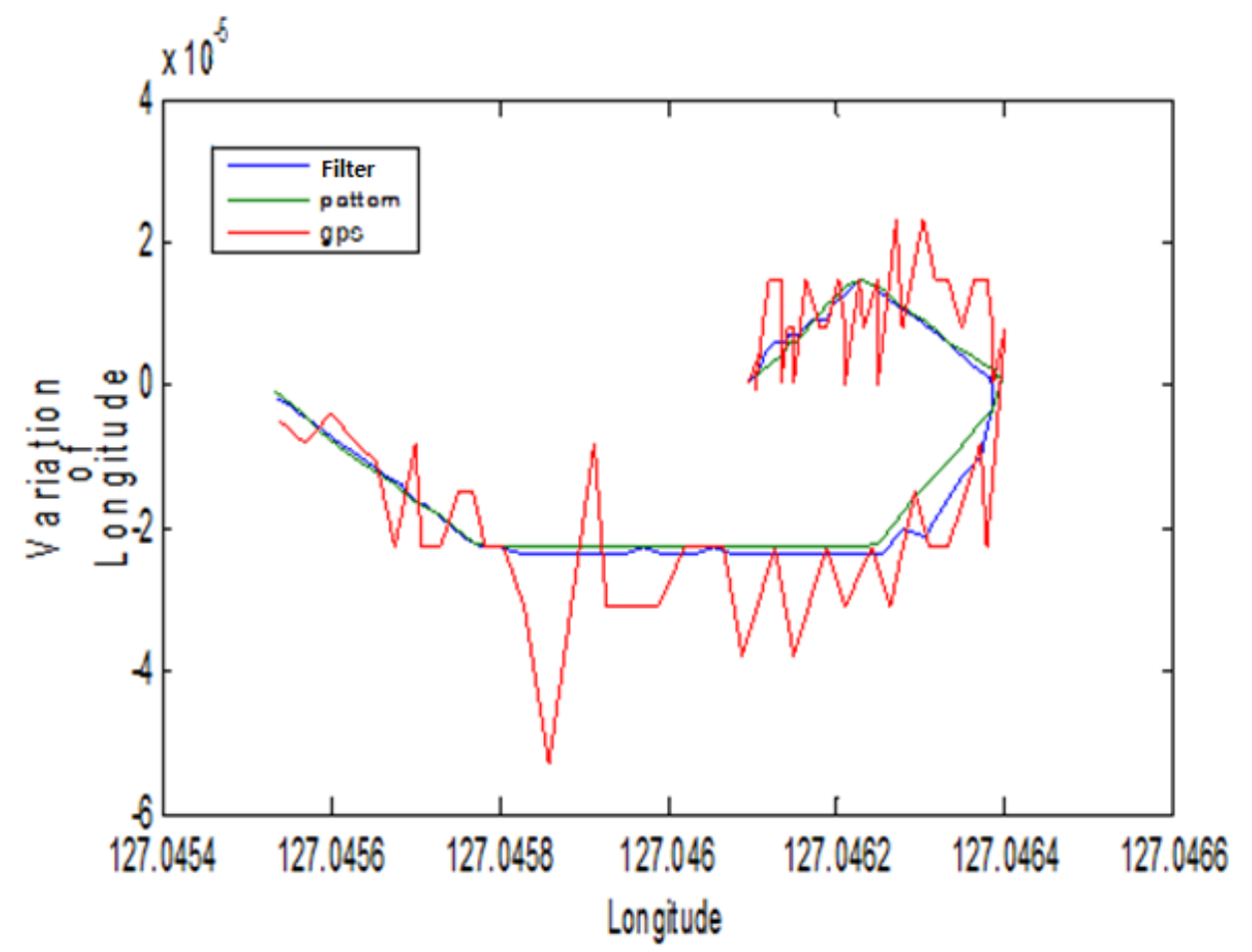

Figure 14. Velocity Results Depending on Longitude Passed through the Kalman Filter

As shown in Figure 12, GPS data has some noise included which leads to a large error of GPS data in Figure 13 and 14. The results of adaptive Kalman filter are obtained by interaction between GPS data and moving pattern. The results corresponding in Figure 13 and 14 are the filter part. The position result estimated by the Kalman filter is finally revised by the map-matching technique. The map-matching technique used in this paper is point-to-curve matching with results shown in Figure 15.

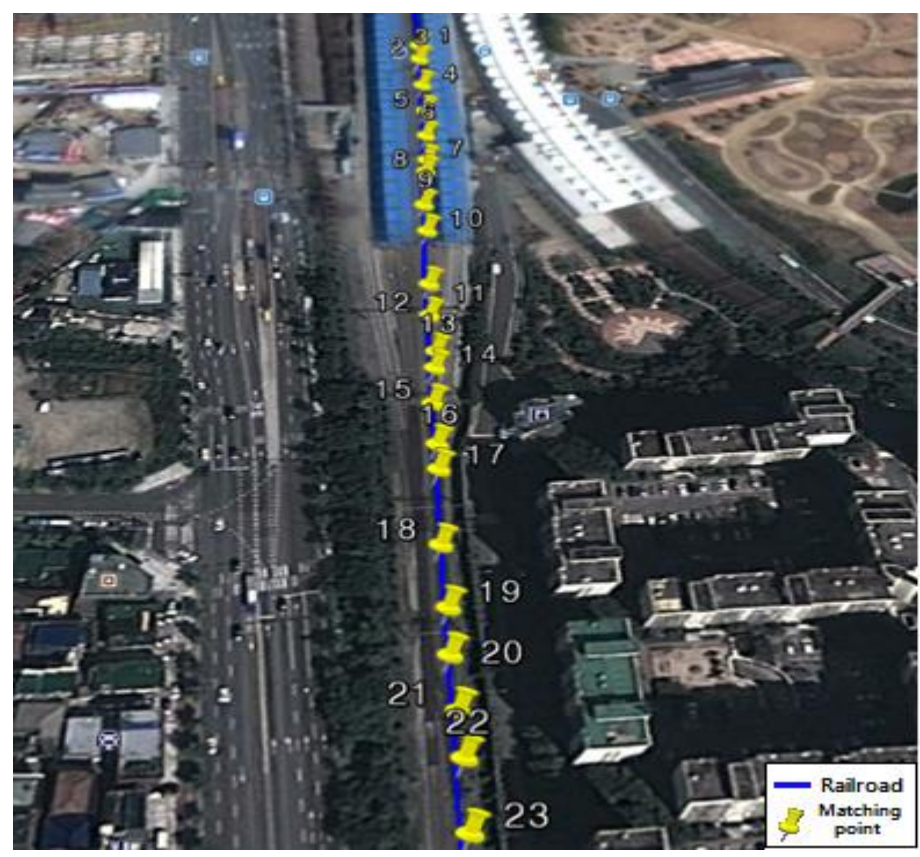

Figure 15. Final Results of Map-matching 


\section{Acknowledgements}

This Research was supported by Seokyeong University in 2012.

\section{References}

[1] J.-S. Yang and S.-P. Kanl, "The Map Matching Algothm og GPS Data”, Journal of the Eastern Asia Society for Transportation Studies, vol. 6, (2005), pp. 2561-2573.

[2] C. E. White, D. Bernstein and A. L. Kornhauser, "Some Map Matching Algorithms for Personal Navigation, Transportation Research Part C", vol. 8, (2000), pp. 91-108.

[3] E. Murakami and D. P. Wagner, "Can using Global Positioning System (GPS) Improve Trip Reporting?", Transportation Research Part C: Emerging Technologies, vol. 7, no. 2-3, (1999), pp. 149165.

[4] S. Brakatsoulas, Dieter Pfoser, Randall Salas and Carola Wenk, "On Map-Matching Vehicle Tracking Data", RA Computer Technology Institute Akteou 11GR-11851 Athens Greece \{sbrakats, pfoser\}@cti.gr, (2005).

[5] J. Greenfeld, "Matching GPS Observations to Locations on a Digital Map", Proc. 81th Annual Meeting of the Transportation Research Board, Washington, DC, (2002).

[6] H. Alt, A. Efrat, G. Rote and C. Wenk, "Matching Planar Maps", J. of Algorithms, vol. 49, (2003), pp. 262-283.

[7] H. Liu, H. Wei, H. Xu and Y. Bao, "Editors Simultaneous Correction of GPS Error and Map Error for Improved Map-matching", Geoinformatics 2008 and Joint Conference on GIS and Built Environment, Proc. of SPIE, vol. 7145, 71452S, (2008).

[8] T. Jo, M. Haseyamai and H. Kitajima, "A Map Matching Method with the Innovation of the Kalman Filtering”, IEICE Trans. Fund. Electron. Comm. Comput. Sci. E79-A, (1996), pp. 1853-1855.

[9] W. Kim, G. Jee and J. Lee, "Efficient Use of Digital Road Map in Various Positioning for ITS", IEEE Symposium on Position Location and Navigation, San Deigo, CA, (2000).

[10] S. K. Honey, W. B. Zavoli, K. A. Milnes, A. C. Philips, M. S. White and G. E. Loughmiller, "Vehicle Navigational System and Method", United States Patent No., 4796191, (1989).

[11] T. Miwa, D. Kiuchi, T. Yamamoto and T. Morikawa, "Development of Map Matching Algorithm for Low Frequency Probe Data, Transportation Research Part C 22, (2012), pp. 132-145.

[12] Y. Cho and H. Choi, "A Position Estimation of Vehicle using Adaptive Kalman Filter based on Moving Pattern”, Advanced Science and Technology Letters, vol.46, Proc. of Int. Workshop,(2014), pp.107-110.

\section{Authors}

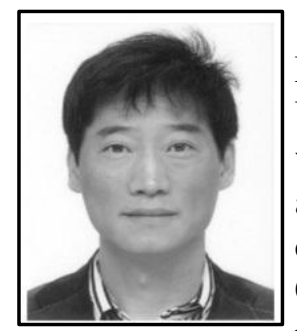

Youngwan Cho was born in Hamyang, Korea, in 1968. He B.S., M.S., and Ph.D. degrees in electronic engineering from Yonsei University, Seoul, Korea, in 1991, 1993, and 1999, respectively. He worked as a Senior Research Engineer in the Control System Group at SAMSUNG Electronics, Seoul, Korea, from 1999 to 2003. He is currently working as an Associate Professor in the Department of Computer Engineering, Seokyeong University, Seoul, Korea. His research interests include fuzzy control theory and applications, nonlinear analysis of intelligent control systems, adaptive and robust control, and robotics and automation.

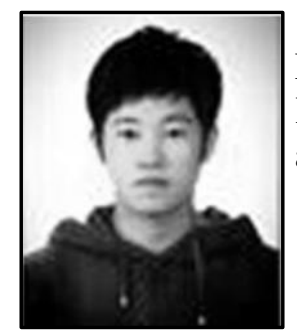

Hyunkyu i was born in Incheon, Korea, in 1987. He received the B.S degree in computer engineering from Seokyeong University, Korea in 2013. He is currently in the master's program of electronic and computer engineering at Seokyeong University. 